\title{
Reliability-Based Phasor Measurement Unit with Outage of Transmission Lines
}

\author{
Muhammad Murtadha Othman ${ }^{1 *}$, Ismail Musirin ${ }^{1}$, Ain Munirah binti Mohd Rosli ${ }^{1}$, Mohammad Lutfi Othman ${ }^{2}$ \\ ${ }^{I}$ Universiti Teknologi MARA, Faculty of Electrical Engineering, 40450 Shah Alam, Selangor, Malaysia \\ ${ }^{2}$ Centre for Advanced Power and Energy Research and Department of Electrical and Electronics Engineering, Universiti Putra \\ Malaysia, Faculty of Engineering \\ *Corresponding author E-mail: mamat505my@yahoo.com
}

\begin{abstract}
This paper discussed on the Monte-Carlo simulation technique to determine the optimal placement of Phasor Measurement Unit (PMU) in power system whilst ensuring the observability of the system. In addition, the information on Force Outage Rate (FOR) of the system can be calculated using Markov Chain technique. The FOR represents the level of risk security for the transmission line that happened because of unscheduled and unexpected failure or repair in the system. Subsequently, the reliability model of the transmission line can be developed. Using IEEE 57-bus system, the results obtained from Monte-Carlo simulation technique demonstrate the optimal PMU placement, the desired reliability of the Wide Area Monitoring System (WAMS) as well as the number and location of covered contingencies of the system.
\end{abstract}

Keywords: Force Outage Rate (FOR); Markov Chain; Monte-Carlo Simulation; Phasor Measurement Unit (PMU).

\section{Introduction}

In recent times, power systems are being operated under heavily stressed conditions because of the increment in electricity demand and competitive market environment. [1] Therefore, the system may be affected by unexpected contingency events. To overcome this problem whilst refining the system in term of monitoring, protection and control, Phasor Measurement Unit (PMU) was invented. PMU is a wide area measurement device in Wide Area Monitoring System (WAMS) that is used for measuring the voltage phasor and current phasor from widely dispersed location. [2] Unlike traditional measurement units, the PMU is able to measure the voltage phasor of the installed bus and the current phasor of all the lines connecting to the bus. This criterion makes the installed bus and its neighboring buses become observable. [3]

In a wide-area power network, it is neither economical nor necessary to install a PMU at each bus. So, the PMU placement problem concerns with the location and number of PMUs that should be implemented to a power system to become observable. [4] Various methodologies have been developed in finding the optimal placement of PMU to overcome this issue. [5] However, the power system reliability must be considered in defining the complete system observability.

The probability that a system will perform its proposed function under pre-specified operating conditions for a period of time is defined as the system reliability. [6] Markov model is one of the common methods used in reliability engineering. There are many researches done regarding Markov model used to analyse the reliability of conventional power system. [7]

The four-state model of transmission line as two component showed in [2] was used as the starting point of this paper. Subsequently, the reliability model of transmission line is developed by using Markov Chain method. The components of the transmission line are arranged in parallel. The Markov Chain is used to calculate the force outage rate, FOR of a transmission line. Then, the FOR is used to estimate the assessment of the system reliability in order to determine the availability and unavailability of the transmission line. In addition, the FOR in transmission line is required for minimizing the PMUs placement in normal operating condition and during contingency events where the system observability is maintained.

By using Monte-Carlo simulation technique to execute the minimisation of PMU placement problem, the objectives of this paper such as to determine the optimal location of PMUs needed for full system observability, to calculate the reliability of the system and to perceive the observability of the system during contingency event can be obtained. The significance of this project is because each bus can be made observable either by its own PMU or by PMUs at adjacent bus. By determining the reliability of the system, the placement of PMUs can be arranged in line with the observability of the system. The scope of this study is to analyse the reliability of IEEE 57-bus system by the optimal location of PMUs and system observability. The reliability of the system will be analyses based on the number and location of PMU placement and number of covered $\mathrm{N}-1$ contingencies.

\section{Methodology}

This section will discuss in-depth on the concept of reliability assessment of transmission line, PMU placement problem and Monte-Carlo simulation technique for PMU placement. 


\subsection{Reliability Assessment of Transmission Line}

In order to select the most reliable buses for PMU placement, transmission line failures must be considered. Transmission line failure is modelled as independent single outage considered only first order contingencies that are not related to other failures that may occur at the same time.

According to the number of buses, transmission line failures can be modelled as a single component which can have either in service (up) mode, out of service (down) mode and two (or more) repairable independent components with dependent outage [2].

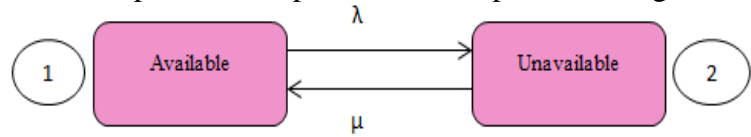

Fig. 1: Model of a transmission line as a single component.

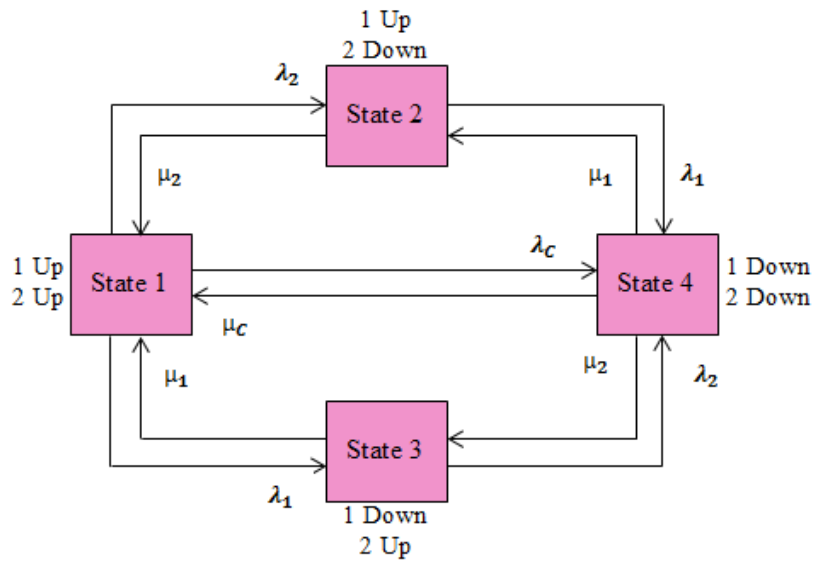

Fig. 2: Model of a transmission line as a two component.

\subsubsection{Determination of Failure Rate and Repair Rate}

In developing the estimation of $\lambda$ and $\mu$, the mean time between failure, MTBF and mean time to repair, MTTR must be calculated first [1].

The mean time between failures, MTBF is expressed by dividing the number of hours in the observation interval by number of random failure. Then, the failure rate is calculated to provide the number of failures that occurs on their component per hour as shown in equation (1).

$\lambda=\frac{1}{M T B F}$

The mean time to repair, MTTR is expressed as the sum of all corrective maintenance divided by the total number of failures during observation interval. In equation (2), the repair rate is calculated to provide the number of occurrence on their component per hour.

$$
\mu=\frac{1}{M T T R}
$$

\subsubsection{Determination of FOR Using Markov Chain}

In this paper, transmission line failures can be modeled as Fourstate Markov Model applied for two components system as shown in Figure 2. Two generators in generating unit are represented in the model. The model uses constant average failure and repair rates.

The basic equation of reliability (availability) of the component can be calculated by using equation (3).

$R=\frac{\mu}{\lambda+\mu}$

There are two methods used in formulating the equation probability of Four-state Markov Model for two components system modeled as transmission line failures. The first method is by using the stochastic transitional probability matrix [8]. This technique is more complex as the equation is hard to be derived from the matrix.

$$
\begin{aligned}
P= & {\left[\begin{array}{llll}
P_{1} & P_{2} & P_{3} & P_{4}
\end{array}\right] \times } \\
& {\left[\begin{array}{cccc}
-\left(\lambda_{1}+\lambda_{2}+\lambda_{\mathrm{C}}\right) & \lambda_{2} & \lambda_{1} & \lambda_{\mathrm{C}} \\
\mu_{2} & -\left(\lambda_{1}+\mu_{2}\right) & 0 & \lambda_{1} \\
\mu_{1} & 0 & -\left(\lambda_{2}+\mu_{1}\right) & \lambda_{2} \\
\mu_{\mathrm{C}} & \mu_{1} & \mu_{2} & -\left(\mu_{1}+\mu_{2}+\mu_{\mathrm{C}}\right)
\end{array}\right] }
\end{aligned}
$$

$P_{1}+P_{2}+P_{3}+P_{4}=1$

After solving all the equations using substitution method, the probabilities of state equation are formulated as:

$P_{1}=\frac{\mu_{1} \mu_{2} \mu_{\mathrm{C}}}{\left(\lambda_{1}+\mu_{1}\right)\left(\lambda_{2}+\mu_{2}\right)\left(\lambda_{\mathrm{C}}+\mu_{\mathrm{C}}\right)}$

$P_{2}=\frac{\lambda_{2} \mu_{1}}{\left(\lambda_{1}+\mu_{1}\right)\left(\lambda_{2}+\mu_{2}\right)}$

$P_{3}=\frac{\lambda_{1} \mu_{2}}{\left(\lambda_{1}+\mu_{1}\right)\left(\lambda_{2}+\mu_{2}\right)}$

$P_{4}=\frac{\lambda_{1} \lambda_{2} \lambda_{\mathrm{C}}}{\left(\lambda_{1}+\mu_{1}\right)\left(\lambda_{2}+\mu_{2}\right)\left(\lambda_{\mathrm{C}}+\mu_{\mathrm{C}}\right)}$

Subsequently, the probability of the state is determined by using the following equations.

$f_{1}=P_{1}\left(\lambda_{1}+\lambda_{2}+\lambda_{\mathrm{C}}\right)$

$f_{2}=P_{2}\left(\lambda_{1}+\mu_{2}\right)$

$f_{3}=P_{3}\left(\mu_{1}+\lambda_{2}\right)$

$f_{4}=P_{4}\left(\mu_{1}+\mu_{2}+\mu_{\mathrm{C}}\right)$

The availability of the system is shown in the probability when the system is operable which are in State 1, State 2 and State 3. However, the system is unavailable in State 4 due to probability of the component that failed in the operation.

In the case of the two component systems as shown in Figure 2, the value of availability and unavailability (FOR) depend on whether the component is in series or parallel system. The availability and unavailability (FOR) for a series system can be calculated by using equation (14).

Availability, $\mathrm{A}=P_{1}$

Unavailability (FOR), $\mathrm{U}=P_{2}+P_{3}+P_{4}$ or $\mathrm{U}=1-\mathrm{A}$

From reliability point of view, a series system is defined as a set of components where all must be working for the system success or only one need to fail for the system failure. The value of availability and unavailability (FOR) is represented for both generators in that system. The availability and unavailability (FOR) for a parallel system can be calculated by using equation (16).

Availability, $\mathrm{A}=P_{1}+P_{2}+P_{3}$

Unavailability (FOR), $\mathrm{U}=P_{4}$ or $\mathrm{U}=1-\mathrm{A}$

From a reliability point of view, a parallel system is defined as a set of components when only one need to work for system success or all must fail for system failure. Same as series system, the value of availability and unavailability (FOR) in parallel system is represented for both generators in the system.

For this case, the reliability model of transmission line is developed by using Markov Chain process and the components are arranged in parallel system. The Markov Chain is used to calculate the force outage rate, FOR of a transmission line. Then, the FOR is used in estimating the assessment of the system reliability. 


\subsection{PMU Placement Problem}

The objective of PMU placement problem is to achieve the availability of the chosen bus and all lines incident to that bus by using minimum number of PMUs. The problem in placing PMUs for $n$ bus system can be formulated as

$\min \sum_{i=1}^{N} x_{i}$

where the components of binary decision variable vector $\left(x_{i}\right)$ are defined as

$x_{i}= \begin{cases}1, & \text { if a PMU is placed at bus } i \\ 0, & \text { otherwise }\end{cases}$

$f_{i}(x)=\sum_{k \in \Omega i}^{N} x_{k} \geq 1$

$f_{i}(x)$ is the constraint of observability for each bus $i$, where the elements involved is the sum of the variable that denote the bus $i$ and the set of variables that denote the buses connected to the bus $i\left(\Omega_{i}\right)$ to ensure the observability of the system at all buses.

Based on the network topology, the PMU must be placed at more reliable buses that the probability of losing observability in the system can be reduced when the constraint is more than one.

\subsection{Monte-Carlo Simulation Technique for PMU Placement}

The Monte-Carlo simulation technique used to determine the placement of PMU is expounded in the following procedure.

a) Randomize the number and placement of PMU on the system. The PMU placement is generated randomly.

b) Filter the PMU located at the same bus. Since the process used in a) sometimes may provide the same location of bus for PMU placement, therefore, the chosen PMUs of the same bus location must be filtered so that the location of PMU on the bus is not repeated.

c) Determine the reliability-based PMU with transmission line outages. Firstly, an index is introduced to represent the probability that the PMU and all of its adjacent lines are available.

$\boldsymbol{P i n}_{P M U_{j}}=R_{P M U} \cdot \prod_{k=1}^{\Omega j} R_{k}$

$R_{P M U}$ is the PMU reliability, $R_{k}$ is the reliability or availability of the $k$ th transmission line and $\Omega j$ is the set of adjacent transmission line at bus $j$.

Then, the probability of normal state representing the system without contingencies, $P_{n s}$ and probability of the $k$ th contingency state, $P_{c s_{k}}$ are calculated.

$P_{n s}=\prod_{k=1}^{N_{L}} R_{k}$

$P_{c s_{k}}=Q_{k} \cdot \prod_{\substack{i=1 \\ i \neq k}}^{N_{L}} R_{i}$

where, $N_{L}$ is the number of transmission lines, $R_{k}$ is the reliability or availability of the $k$ th transmission line and $Q_{k}$ is the unreliability (unavailability) of the transmission line $k$.

A state is defined by listing the successful and failed transmission lines in the system. Based on the states of the transmission lines, the probability of occurrence of each successful state is calculated as the summation results in the reliability of the WAMS. The WAMS reliability $\left(R_{w}\right)$ can be calculated as follows:

1) the system is observable under normal state, the WAMS reliability is $P_{n s}$.

2) the system is observable under normal state, and it is still observable under $k$ th contingency state, the WAMS reliability is $P_{n s}+P_{c s_{k}}$.

Next, the system observability is determined using $y_{k}$ line, where $y_{k}$ is the binary decision variable that represents observability of the system under $k$ th contingency state (single line outage $\mathrm{k}$ ). At the transmission lines concerning the PMU placement, $y_{k}=1$ means that the system observability is not affected by contingency of line $k$ and $y_{k}=0$ means that full system observability is not achieved under this contingency.

Then, the total probability of the $k$ th contingency state, $P c s_{k_{-}}$total is calculated where it is the product of each line probability of the $k$ th contingency state, $P c s_{k}$ with $y_{k}=1$.

To improve the WAMS reliability, actual problem model in equation (1) will be modified to include the maximisation of the reliability criterion where

$\sum_{k=1}^{N_{L}}-P C s_{k} \cdot y_{k}$

As PMU placement problem concerns with minimum number of $\mathrm{PMU}, \mathrm{PCs}_{k}$ is introduced with a negative sign to indicate minimisation problem. The objective function will be minimized when more $y_{k}$ is equal to one. The solution then will include $N-1$ contingencies with highest value of $P c s_{k}$.

The reliability-based PMU placement considering transmission line outages has been formulated as an integer linear programming problem in equation (25). In this problem, the objectives include the minimization of the number of PMUs and maximization of the reliability criterion while satisfying the constraint.

$\min \left[\sum_{j=1}^{N}\left(1+Q_{P M U_{j}}\right) \cdot x_{j}-\sum_{k=1}^{N_{L}}-P C s_{k} \cdot y_{k}\right]$

Meanwhile, the constraint in equation (26) ensures that the summation the available lines of the chosen PMUs must exceed zero for the PMUs to be available.

$f_{c}=x_{j} \cdot \sum_{k=1}^{N_{L}} y_{k} \geq 0$

\section{Results and Discussion}

The proposed method in this paper is programmed using Matlab R2013a and Monte-Carlo simulation technique is used to solve the PMU placement problem. The model is solved by using IEEE 57bus system shown in Figure 3 that consists of 80 transmission lines. [2, 9] The IEEE 57-bus system consists of 57 buses, 7 generators and 47 loads. [10]. By referring to [2], the $R_{P M U}$ of IEEE 57-bus system is fixed as 0.995498 . The reliability data for IEEE 57-bus system can be obtained from [11].

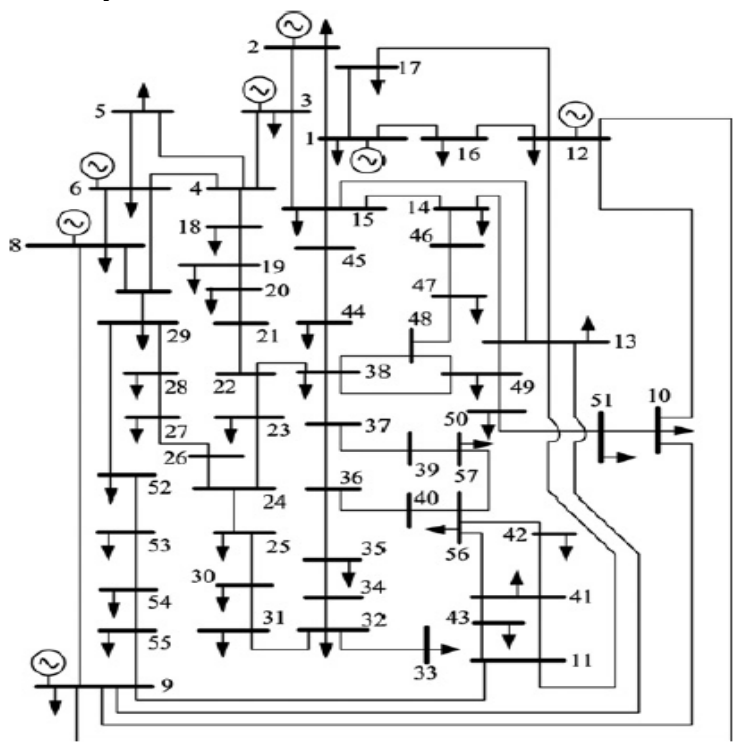

Fig. 3: IEEE 57-bus system.

By using equation (18) and referring to the availability information of transmission lines in [11], the $P_{n s}=68.14 \%$ is calculated for reliability of WAMS that consists of the probability of occurrence of each successful state when system is observable under normal state. 
The summation for occurrence probability of all $\mathrm{N}-1$ contingency state $P_{C S}$ is calculated as $26.21 \%$ according to Table 1 . Hence, the reliability of WAMS $\left(R_{w}\right)$ in the system is calculated as $94.35 \%$. From Table 1, the $P c s_{k}=0.5556$ at lines 18-19 and 34-35 have the highest probability to fail and will become unobservable. Thus, the buses that are related to these lines are considered to be high in priority to be included in PMU placement. Moreover, the reliability of the system is more valid when the PMU is installed at buses where the system is observable under the most probable contingencies. In short, placing the PMUs is preferable at those buses since it will make the system to be fully observable under the most probable contingencies.

Table 2 shows the location of PMUs and the number of covered contingencies after solving the model using equation (25). The results show that when the desired reliability of WAMS $\left(R_{w d}\right)$ increase, the required number of PMUs also increase as well as the number of covered $N-1$ contingencies. The number of covered $N-1$ contingencies indicates the total of available lines when PMU is placed at selected bus so that the system is observable. The high number of covered $\mathrm{N}-1$ contingencies means that the observability of the system is high thus, making the system become more reliable.

Table 1: Probability of N-1 Contingency States for IEEE 57-bus system, $\mathrm{PCS}_{k}$

\begin{tabular}{|c|c|c|c|c|c|c|c|c|}
\hline \multirow{2}{*}{ No. } & \multicolumn{2}{|c|}{ Line } & \multirow{2}{*}{$P c s_{k}$} & \multirow{2}{*}{ No. } & \multicolumn{3}{|c|}{ Line } & \multirow[b]{2}{*}{$P c s_{k}$} \\
\hline & From & To & & & & & To & \\
\hline 1 & 1 & 2 & 0.00274 & 41 & 7 & 29 & & 0356 \\
\hline 2 & 2 & 3 & 0.00384 & 42 & 25 & $\overline{30}$ & & 0322 \\
\hline 3 & 3 & $\overline{4}$ & 0.00164 & 43 & 30 & 31 & & 0515 \\
\hline 4 & 4 & 5 & 0.00529 & 44 & 31 & 32 & & 0349 \\
\hline 5 & 4 & 6 & 0.00515 & 45 & 32 & 33 & & 0549 \\
\hline
\end{tabular}

\begin{tabular}{|l|l|l|l|l|l|l|l|}
\hline 5 & 4 & 6 & 0.00515 & 45 & 32 & 33 & 0.00549 \\
\hline 6 & 6 & 7 & 0.00487 & 46 & 34 & 32 & 0.00404 \\
\hline 7 & 6 & 8 & 0.00232 & 47 & 34 & 35 & 0.00556 \\
\hline 8 & 8 & 9 & 0.00384 & 48 & 35 & 36 & 0.00226 \\
\hline & 9 & 10 & 0.00123 & 49 & 36 & 37 & 0.00425 \\
\hline
\end{tabular}

\begin{tabular}{|c|c|c|c|c|c|c|c|}
\hline 8 & 8 & 9 & 0.00384 & 48 & 35 & 36 & 0.00226 \\
\hline 9 & 9 & 10 & 0.00123 & 49 & 36 & 37 & 0.00425 \\
\hline 10 & 9 & 11 & 0.00308 & 50 & 37 & 38 & 0.00246 \\
\hline
\end{tabular}

\begin{tabular}{|l|l|l|l|l|l|l|l|}
\hline 10 & 9 & 11 & 0.00308 & 50 & 37 & 38 & 0.00246 \\
\hline 11 & 9 & 12 & 0.00260 & 51 & 37 & 39 & 0.00418 \\
\hline 12 & 9 & 13 & 0.00184 & 52 & 36 & 40 & 0.00432 \\
\hline
\end{tabular}

\begin{tabular}{|c|c|c|c|c|c|c|c|}
\hline 12 & 9 & 13 & 0.00184 & 52 & 36 & 40 & 0.00432 \\
\hline 13 & 13 & 14 & 0.00198 & 53 & 22 & 38 & 0.00143 \\
\hline
\end{tabular}

\begin{tabular}{|l|l|l|l|l|l|l|l|}
\hline 13 & 13 & 14 & 0.00198 & 53 & 22 & 38 & 0.00143 \\
\hline 14 & 13 & 15 & 0.00308 & 54 & 11 & 41 & 0.00232 \\
\hline
\end{tabular}

\begin{tabular}{|l|l|l|l|l|l|l|l|}
\hline 15 & 1 & 15 & 0.00157 & 55 & 41 & 42 & 0.00219 \\
\hline 16 & 1 & 16 & 0.00391 & 56 & 41 & 43 & 0.00418 \\
\hline 17 & 1 & 17 & 0.00329 & 57 & 38 & 44 & 0.00501 \\
\hline
\end{tabular}

\begin{tabular}{|l|l|l|l|l|l|l|l|}
\hline 16 & 1 & 16 & 0.00391 & 56 & 41 & 43 & 0.00418 \\
\hline 17 & 1 & 17 & 0.00329 & 57 & 38 & 44 & 0.00501 \\
\hline 18 & 3 & 15 & 0.00432 & 58 & 15 & 45 & 0.00274 \\
\hline
\end{tabular}

\begin{tabular}{|l|l|l|l|l|l|l|l|}
\hline 18 & 3 & 15 & 0.00432 & 58 & 15 & 45 & 0.00274 \\
\hline 19 & 4 & 18 & 0.00432 & 59 & 14 & 46 & 0.00473 \\
\hline
\end{tabular}

\begin{tabular}{|l|l|l|l|l|l|l|l|}
\hline 19 & 4 & 18 & 0.00404 & 60 & 46 & 47 & 0.00425 \\
\hline
\end{tabular}

\begin{tabular}{|c|c|c|c|c|c|c|c|}
\hline 21 & 5 & 6 & 0.00130 & 61 & 47 & 48 & 0.00116 \\
\hline 22 & 7 & 8 & 0.00143 & 62 & 48 & 49 & 0.00391 \\
\hline
\end{tabular}

\begin{tabular}{|c|c|c|c|c|c|c|c|}
\hline 22 & 7 & 8 & 0.00143 & 62 & 48 & 49 & 0.00391 \\
\hline 23 & 10 & 12 & 0.00260 & 63 & 49 & 50 & 0.00287 \\
\hline
\end{tabular}

\begin{tabular}{|l|l|l|l|l|l|l|l|}
\hline 23 & 10 & 12 & 0.00260 & 63 & 49 & 50 & 0.00287 \\
\hline 24 & 11 & 13 & 0.00356 & 64 & 50 & 51 & 0.00536 \\
\hline
\end{tabular}

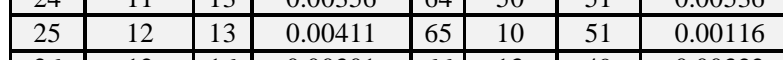

\begin{tabular}{|l|l|l|l|l|l|l|l|}
\hline 26 & 12 & 16 & 0.00301 & 66 & 13 & 49 & 0.00322 \\
\hline 27 & 12 & 17 & 0.00487 & 67 & 29 & 52 & 0.00308 \\
\hline
\end{tabular}

\begin{tabular}{|l|l|l|l|l|l|l|l|}
\hline 26 & 12 & 16 & 0.00301 & 66 & 13 & 49 & 0.00322 \\
\hline 27 & 12 & 17 & 0.00487 & 67 & 29 & 52 & 0.00308 \\
\hline
\end{tabular}

\begin{tabular}{|l|l|l|l|l|l|l|l|}
\hline 28 & 14 & 15 & 0.00446 & 68 & 52 & 53 & 0.00322 \\
\hline 29 & 18 & 19 & 0.00556 & 69 & 53 & 54 & 0.00467 \\
\hline
\end{tabular}

\begin{tabular}{|l|l|l|l|l|l|l|l|}
\hline 29 & 18 & 19 & 0.00556 & 69 & 53 & 54 & 0.00467 \\
\hline
\end{tabular}

\begin{tabular}{|l|c|c|c|c|c|c|c|}
\hline 30 & 19 & 20 & 0.00356 & 70 & 54 & 55 & 0.00260 \\
\hline 31 & 21 & 20 & 0.0026 & 71 & 11 & 43 & 0.00473 \\
\hline
\end{tabular}

\begin{tabular}{|l|l|l|l|l|l|l|l|}
\hline 31 & 21 & 20 & 0.0026 & 71 & 11 & 43 & 0.00473 \\
\hline
\end{tabular}

\begin{tabular}{|l|l|l|l|l|l|l|l|}
\hline 32 & 21 & 22 & 0.00164 & 72 & 44 & 45 & 0.00329 \\
\hline 33 & 22 & 23 & 0.00391 & 73 & 40 & 56 & 0.00130 \\
\hline
\end{tabular}

\begin{tabular}{|l|l|l|l|l|l|l|l|}
\hline 33 & 22 & 23 & 0.00391 & 73 & 40 & 56 & 0.00130 \\
\hline 34 & 23 & 24 & 0.00473 & 74 & 56 & 41 & 0.00191 \\
\hline
\end{tabular}

\begin{tabular}{|l|l|l|l|l|l|l|l|}
\hline 34 & 23 & 24 & 0.00473 & 74 & 56 & 41 & 0.00191 \\
\hline 35 & 24 & 25 & 0.00308 & 75 & 56 & 42 & 0.00446 \\
\hline 36 & 24 & 25 & 0.00157 & 76 & 39 & 57 & 0.00329 \\
\hline 37 & 24 & 26 & 0.00232 & 77 & 57 & 56 & 0.00184 \\
\hline 38 & 26 & 27 & 0.00184 & 78 & 38 & 49 & 0.00267 \\
\hline 39 & 27 & 28 & 0.00239 & 79 & 38 & 48 & 0.00391 \\
\hline 40 & 28 & 29 & 0.00315 & 80 & 9 & 55 & 0.00198 \\
\hline
\end{tabular}

Table 2: Reliability-based PMU Placement at IEEE 57-bus System

\begin{tabular}{|c|c|c|c|}
\hline$R_{w d, \%}$ & $\begin{array}{c}\text { No of } \\
\text { PMUs }\end{array}$ & PMU location & $\begin{array}{c}\text { Covered } N-1 \text { Contin- } \\
\text { gencies }\end{array}$ \\
\hline
\end{tabular}

\begin{tabular}{|c|c|c|c|}
\hline 76.80 & 10 & $3,4,5,6,8,11,13,16,17,18$ & 29 \\
\hline 77.54 & 18 & $\begin{array}{c}1,3,6,7,8,9,10,12,13,15,1 \\
6,19\end{array}$ & 40 \\
\hline 78.29 & 21 & $\begin{array}{c}5,9,10,11,12,14,16,17,18 \\
20,21,22,23,24,25,27,29, \\
31,32,35,37\end{array}$ & 49 \\
& & $\begin{array}{c}2,3,6,7,8,9,10,11,13,14, \\
15,16,17,18,19,21,23,24, \\
25,27,30,31,32,33,34,35, \\
36,38,40,44,45,46,48,49, \\
53,54\end{array}$ & 59 \\
\hline 80.08 & 36 & \\
& & \begin{tabular}{c} 
\\
\hline
\end{tabular}
\end{tabular}

$R_{w d}$ is the summation of the probabilities of the observable states by the WAMS. The increasing in $R_{w d}$ will enhance the monitoring system capability when facing possible transmission line outages. Hence, a high $R_{w d}$ implies that system will be observable under several $N-1$ contingencies (high-probability contingencies). The system would not be observable under contingencies with low probability when $R_{w d}$ decreases.

According to Table 3, the increasing in number of covered $N-1$ contingencies ensures that the system will be more observable as the number of available transmission lines increases. The line of $18-19$ or $34-35$ is always included to make sure that the system can achieve full observability.

According to the case study in constraint equation (26), all chosen PMUs can be used since the values that we get are more than 0 . When the constraint condition is achieved, it means that the line is observable thus; the system observability will be achieved under this parameters.

Table 3: The Observability of the IEEE 57-bus System according to the Reliability-based PMU Placement

\begin{tabular}{|c|c|c|c|}
\hline$R_{w d, \%}$ & $\begin{array}{l}\text { No of } \\
\text { PMUs }\end{array}$ & $\begin{array}{c}\text { Covered N-1 } \\
\text { Contingencies }\end{array}$ & $\begin{array}{l}\text { Lines Attached to the Buses that } \\
\text { Installed PMUs }\end{array}$ \\
\hline 76.80 & 10 & 29 & $\begin{array}{l}6-7,6-8,8-9,9-10,9-11,9-13, \\
13-14,3-15,4-18,7-8,14-15, \\
18-19,19-20,21-20,21-22,22- \\
23,28-29,7-29,25-30,30-31, \\
36-37,36-40,11-41,41-43,15- \\
45,47-48,48-49,13-49,52-53\end{array}$ \\
\hline 77.54 & 18 & 40 & $\begin{array}{l}1-2,2-3,3-4,6-7,6-8,9-11,9- \\
12,9-13,13-14,13-15,1-15,1- \\
16,1-17,4-18,5-6,7-8,11-13, \\
12-13,12-16,12-17,14-15,18- \\
19,23-24,24-25,28-29,30-31, \\
31-32,34-32,34-35,35-36,36- \\
37,37-38,37-39,22-38,41-42, \\
41-43,15-45,47-48,48-49,29- \\
52\end{array}$ \\
\hline 78.29 & 21 & 49 & $\begin{array}{l}13-15,1-15,1-16,1-17,3-15,4- \\
18,4-18,5-6,10-12,14-15,18- \\
19,19-20,21-20,21-22,22-23, \\
24-25,24-26,26-27,31-32,32- \\
33,34-32,34-35,35-36,37-38, \\
37-39,36-40,22-38,11-41,41- \\
42,38-44,14-46,46-47,47-48 \\
48-49,49-50,50-51,10-51,13- \\
49,52-53,53-54,54-55,11-43 \\
44-45,40-56,56-41,56-42,39- \\
57\end{array}$ \\
\hline 80.08 & 36 & 59 & $\begin{array}{l}4-5,4-6,6-7,6-8,9-11,9-12,9- \\
13,13-14,13-15,1-15,1-16,1- \\
17,3-15,4-18,7-8,10-12,11- \\
13,12-13,12-16,12-17,14-15, \\
18-19,19-20,21-20,21-22,22- \\
23,23-24,24-25,26-27,27-28, \\
28-29,30-31,31-32,32-33,34- \\
32,34-35,35-36,36-37,37-38, \\
37-39,36-40,11-41,41-43,38- \\
44,15-45,14-46,46-47,47-48, \\
48-49,49-50,50-51,10-51,13- \\
49,29-52,52-53,54-55,11-43, \\
56-41,56-42\end{array}$ \\
\hline
\end{tabular}




\section{Conclusion}

This paper proposes a new modified formulation for reliabilitybased Phasor Measurement Unit problem where transmission line outages are taken into account. The method also accounts for availabilities of transmission line condition as the constraint. From this paper, the desired reliability of WAMS, number and location of PMUs as well as number of covered $\mathrm{N}-1$ contingencies can be obtained by developing the reliability objective function as binary linear integer problem in Monte-Carlo simulation technique.

\section{Acknowledgement}

This authors would like to acknowledge the Institute of Research Management \& Innovation (IRMI), UiTM Shah Alam, Selangor, Malaysia for the financial support of this research. This research is supported by IRMI under the LESTARI Research Grant Scheme with project code : 600-IRMI/MyRA5/3/LESTARI (0024/2016).

\section{References}

[1] Chakrabarti S, Kyriakides E \& Eliades D (2009), Placement of synchronized measurements for power system observability. IEEE Transactions on Power Delivery, 24, 12-19.

[2] Gomez O, Anders G \& Rios M (2014), Reliability-based phasor measurement unit placement in power systems considering transmission line outages and channel limits. IET Generation, Transmission and Distribution, 8, 121-130.

[3] Gou B (2008), Generalized integer linear programming formulation for optimal PMU placement. IEEE Transactions on Power System, 23, 1099-1104.

[4] Aminifar F, Lucas C, Khodaei A \& Fotuhi-Firuzabad M (2009), Optimal placement of phasor measurement units using immunity genetic algorithm. IEEE Transactions On Power Delivery, 24, 1014-1020.

[5] Manousakis NM, Korres GN \& Georgilakis PS (2011), Optimal placement of phasor measurement units: a literature review. 16th International Conference on Intelligent System Application to Power Systems (ISAP), 1-6.

[6] (1990), A Compilation of IEEE Standard Computer Glossaries. IEEE Standard Computer Dictionary.

[7] Albuquerque R \& Paucar V (2013), Evaluation of the PMUs measurement channels availability for observability analysis. IEEE Transactions on Power System, 28, 2536-2544.

[8] Ramakumar R (1993), Engineering Reliability Fundamentals and Applications. Prentice-Hall International Editions, pp.239-249.

[9] Aminifar F, Fotuhi-Firuzabad M, Shahidehpour M \& Khodaei A (2011), Observability enhancement by optimal PMU placement considering random power system outages. Energy System, 2, 4565.

[10] IEEE 57-Bus System Illino is Center for a Smarter Electric Grid (ICSEG)", Icseg.iti.illinois.edu, 2016. [Online]. Available : http://icseg.iti.illinois.edu/ieee-57-bus-system/. [Accessed: 07-Jun2016].

[11] Putranto LM, Hara R, Kita H \& Tanaka E (2014), Voltage stabilitybased PMU placement considering $N$-1 line contingency and power system reliability. The 2nd IEEE Conference on Power Engineering and Renewable Energy, 120-125. 